\title{
11. Eine Tetrakoralle aus Yamanba Kalkschicht, Sakawa Gegend, Sikoku, Japan.
}

\author{
Von Masao Mixato. \\ (Comm. by H. YABE, M.J.A., Feb. 19, 1949.)
}

Bis jetzt war die Yamanba Kalkschicht, Sakawa Gegend, Shikoku palaeontologisch wenig erforscht worden. Larin waren nur die folgenden Fossilien bekannt geworden.

Omphalophyllia yamanbaensis YABE et SUGIYAMA

Parafusulina sp.

Schizophoria indica (WAAGEN)

Schizophoria sp.

Oithotichia derbyi (WAAGEN)

Camarophoria mutabilis TsCHERXYsCHEW

Streptorphynchus pseudopelargonatus (BROILI)

Teekella? sp.

Aulosteges polyangensis (KAYSER)

Squamularia asiatica CHAO

Martinia orbicularis GEMMELLARO

Spiriferina multiplicata SOWERBY

Spiriferina nasuta WAAGEN var.

Spiriferina sp.

Der Verfasser hat neuerdings auch dort eine Fossiliensammlung gemacht, dabei hat er eine Art von Genus Lonsdaleiastraea gefunden welche einer neuen Spezies angehoerig ist.

Solche Gattungen wie Lonsdaleiastraea GERTH 1927, Wentzelella GRABAU 1927, Protolonsdaleiastraea Gonsky 1931 und Wentzelloides YABE et MIrato 1944 sind beinahe dicselbe in der zu Grunde liegenden Struktur der Korallite, man sieht aber dabei das fortschreitende Verschwinden der Aussenwände von Wentzelella-Wentzelloides bzw. Protolonsdaleiastraea bis zur Lonsdaleiastraea. Am Genus Lonedleiastraea ist die Spezialisation in dieser Richtung am vollkommensten durchgeführt'

Die neue spezies aus der Yamanba Kalkschicht steht an L. typica GERTH aus Kyam im östlichen Karakorum, Himalaya sehr nahe, weicht aber etwas davon ab. Der Verfasser möcht sie L. yamanbaensis heissen.

Noch eine andere neue japanische Spezies von Lonsdaleiastraea hatte ich 
schon früle: in der Sammlung des zu früh verstorbenen Professors Y. OzAWA gefunden, die ich mit freundlicher Erlaubnis von Prof. T. KOBAYASHI dem jetzigem Besitzer untersuchen konnte. Diese neue, von mir L. nipponica benannte kommt in der Yabeina Kalkschicht in der Gegend von Shiraiwa, Provinz Nagato, vor zusammen mit Yabeina shiraiwensis OZAWA, Neoschwagerina margaritae DEPRAT, N. craticulifera SCHWAGER, Waagenophyllum akasakensis (YABE), Wentzelella subtimorica HUANG, und Lonsdaleia katoi OzAWA.

Die Art von Nagato ist in keiner Weise mit $L$. yamanbaensis vergleichbar, wie auch die permischen Lonsdaleiastraea Schichten bei Nagato mit der Yamanba Kalkschicht stratigraphisch nicht direkt zur Vergleichung bringen kann. Aber Lonsdaleiastraea typica, welche am nächsten der neuen japanischen Spezies

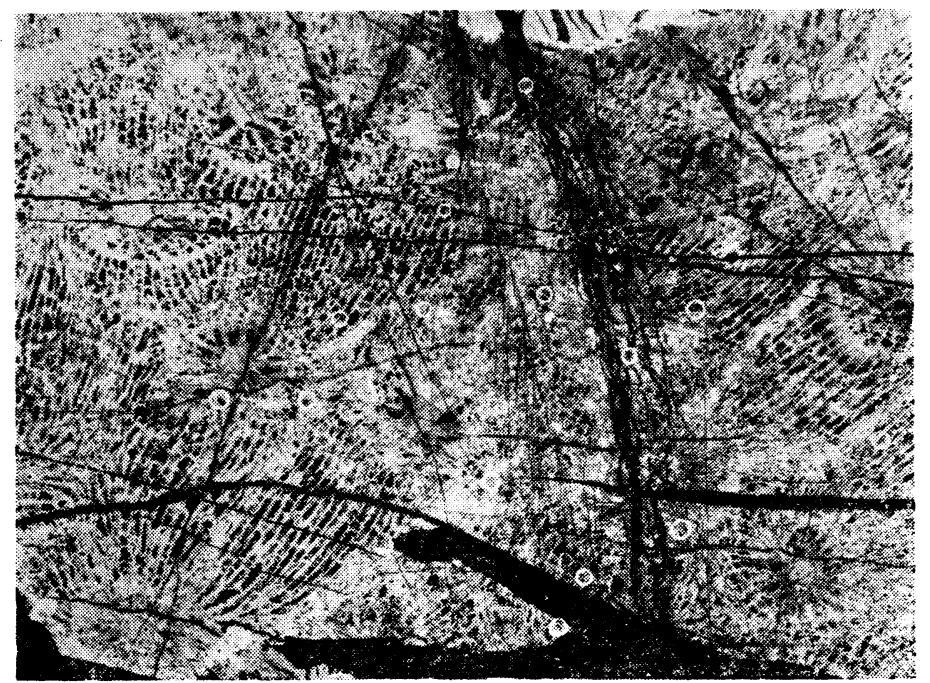

Fig. 1. $\times$ Ca. 6 .

Lonsdaleiastraea yamanbaensis MiNaTo, sp. nov.

L. yamanbaensis zu stehen scheint, kommt zusammen mit solchen Korallen wie Waagenophyllum indicum, Wentzelella caracorumensis und Stylidophyllum variabite vor. Man kann deshalb indirekt schliessen, dass stratigraphisch die Yamanba Kalkschicht auch zum Mittelperm zu stellen ist. Schon J. NoNAKA, der die Brachiopoden-Fauna der Yamanba Kalkschicht früher untersucht hatte, war der Ansicht dass die darin vorkommenden Brachiopoden teils mit dennen der Unter bzw. Mittel Productus Kalkschcht von ,Salt Range," und teils mit denen von Guadaloupe, vergleichbar sind und deshalb die Yamanba Kalkschicht dem Nittetperm zu gehörig sei. Der Verfasser stimmt ihm in dieser Hinsicht vollkommen ein. Die Yamanba Fauna zeigt höchst wahrscheinlich die Yabeina Stufe des japanischen Mittelperm (Kanokura Serie). 
Die Koralle steckt ganz im Gestein, und ihre Aussenseiten sind schwer zu beobachten. Die Beschreibung deshalb ist nur an Dünnschliffen möglich. Die Koralliten schliessen sich eng aneinander, uud die Aussenwände sind beinähe vollkommen verschwunden. Die Septen bei benachbarten Koralliten sind vollkommen verbunden und zeigen eine Thlımnastraea-ähnltche Anordnung. Es gibt drei Arten Septen: I. Ordnung, lang und durch Stereoplasma verdickt: II. Ordnung, etwas kürzer und dünner: III. Ordnung noch kürzer, dabei zeigen sie sich einen etwas unvollständigen Zyklus. Das Säulchen ist klein, einfach gebaut, steht teilweise in Verbindung mit einem Septum der I. Ordnung und meistens ist durch Stereoplasma sehr verdickt; niemals kann man deutliche Medianplatten in Querschnitten erkennen, aber Andeutungen davon sind im Längsschliffe sichtbar. Der Raum zwischen den Septen ist mit zahlreichen konzentrischen Dissepimenten. ausgefüllt.

L. typica aus dem östlichen Karakorum ist der vorliegenden Art besonders nahe stehend; aber bei der letzteren sind die Septen der I. Ordnung dicker und länger als die der II. Ordnung, während die Verschiedenheit der Septen der I. und II. Ordnung bei der GER'TH-Spezies viel undeutlicher ist.

Bibliographie.

H. YABE: und T. Sugryama: A new form of the Omphalophyllia collected from Yamanba, near Sakawa-machi, Province of Tosa (Shikoku), Jap. Jour. Geol. Geogr., vol. X, 1933, pp. 112-114.

H. GERTH: Permokorallen aus dem Oestlichen Karakorum und Triaskorallen aus dem Nordwestlichen Himalaya. Palaeontographica 88 A. 1938, p. 232, Taf. XV, fig. 7. M. Minato: Carboniferous and Permian Corals of Japan, im Druck. 